\title{
Trabajo en grupo aplicado para la realización de las memorias de prácticas de una asignatura de corte jurídico para ingenieros.
}

\section{Juan Vicente Oltra Gutiérrez}

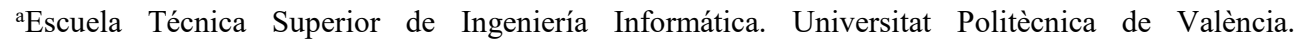
jvoltra@omp.upv.es

\begin{abstract}
In the present work we tell the results of an experience in a legal subject, very different in content to the rest of the subjects with which it shares a degree, focused on changing the way of working in the practices, which, if carried out individually, they happen to be made in group. Both the perceptions of the student and the objective data on the delay of the deliveries are valued, as well as the obstacles that the teacher encounters during the performance of the same.
\end{abstract}

Keywords: group work, methodology, training.

\begin{abstract}
Resumen
En el presente trabajo se exponen los resultados de una experiencia realizada en una asignatura de corte jurídico, muy distinta en contenidos al resto de las asignaturas con las que comparte grado, centrada en el cambio del modo de trabajo en las prácticas de la asignatura, que, de realizarse individualmente, pasan a hacerse en grupo. Se valoran tanto las percepciones del alumno como los datos objetivos en la demora de las entregas, así como los obstáculos que el docente encuentra durante la realización de la misma.
\end{abstract}

Palabras clave: trabajo en grupo, metodología, formación.

\section{Introducción}

En el presente trabajo se expone una experiencia basada en la creación de grupos de trabajo para la realización de las prácticas de una asignatura con una serie de elementos diferenciales con respecto al resto de las de su titulación que acarraba como efecto unos resultados académicos que no eran acordes al montante global.

La asignatura, con un marcado carácter jurídico, es la única de ese corte que se imparte en el Grado de Ingeniería Informática de la Universitat Politècnica de València, en el que el alumno típicamente se encuentra con materias de aplicación inmediata $\mathrm{o}$, al menos, con una cercanía a la creación de código informático más directo. 
Trabajo en grupo aplicado para la realización de las memorias de prácticas de una asignatura de corte jurídico para ingenieros.

Esto generaba entre otros efectos alumnos que costaba recuperar para el ritmo normal, al quedar su atención dispersa ante elementos que percibía como ajenos y, por consiguiente, retrasos en las entregas de las memorias de las prácticas.

La escasa duración de cada sesión de prácticas (90 minutos) y el elevado número de asistentes a cada sesión (dependiendo del grupo, de 20 a 40 alumnos) impedía una atención personalizada del profesor, siendo los retrasos cada vez más numerosos y grandes en el tiempo.

Apoyándonos en una experiencia previa en las sesiones de teoría (Oltra, 2018), donde compartíamos un problema similar al trabajar con casos de debate en aula y tratarse de grupos muy numerosos, que impedía la participación individual de todos los alumnos tratamos de aplicar mecanismos similares en el aula de prácticas donde, además, existía el elemento adicional de una memoria que se ha de entregar.

\section{Objetivos}

El principal objetivo es evaluar de que modo el trabajo en grupo en las practices de una asignatura de corte jurídico impartida a ingenieros consigue reducir el número de alumnos que, o bien se retrasan en las entregas, o bien directamente se descabalgan de la asignatura, frente a la experiencia de cursos anteriores donde la entrega era individual. La media de cursos precedentes, donde no se aplicaba el trabajo en grupo durante las prácticas, alcanzaba un $10 \%$ de alumnos que no llegaban a realizar alguna entrega y un $30 \%$ que entregaba al menos una de ellas con retraso.

La principal dificultad estribó precisamente en la conversion de las practices, no solo por lo que supone de rediseño y elaboración de actividades nunca realizadas en la asgnatura, sino por la evangelización sobre sus bondades en el colectivo de profesores (al tratarse de una asignatura con muchos grupos de practices, es natural pensar que muchos docents estaban muy acostumbrados a la realización clásica de forma individual y tuvieron que salir de lo que denominamos su "zona de confoort".

El problema más evidente, al margen de lo expuesto en el párrafo anterior, que surge de la suma de los factores de la masificación en el laboratorio junto con la escasa sintonía de los contenidos con los de otras asignaturas de la titulación, ha quedado ya enunciado.

El elevado número de asistentes, conjugado con el reducido tiempo que dispone el profesor por alumno, se matiza de alguna manera al formar grupos, pues se reduce por una parte el tiempo dedicado a las consultas, pues se realizan en común para el grupo y, además, siempre existe la posibilidad de que un miembro del grupo, con un grado de comprensión mayor que el del resto, ejerza de primus interpares y aclare las dudas a sus compañeros, mientras el profesor atiende a otro grupo.

En cuanto a los contenidos particulares de las sesiones, que incluyen elementos necesarios para su formación pero poco o nada vistos en el resto de la titulación, como puede ser la búsqueda sentencias en la base de datos Aranzadi, de legislación nacional o europea en los distintos buscadores legales, de normas técnicas en AENOR, de recursos de diversa índole en la Agencia Española de Protección de Datos, e incluso la redacción de documentos de 
cierta complejidad, como breves dictámenes periciales, si bien con el paso del tiempo ha ido siendo paliado mediante la grabación de pequeños vídeos tutoriales, el hecho de poder realizar en equipo esa serie de actividades provoca que se agilice el aprendizaje de las mismas, como tendremos ocasión de comprobar mediante una pequeña encuesta que se les pasó a los alumnos al finalizar la docencia de la asignatura.

En concreto intentamos, dada la nueva estrategia a seguir:

- Medir la mejora en la tasa de retrasos y de ausencias en las entregas de las memorias

- Recoger la opinión apriorística del alumnado, que ya ha cursado asignaturas donde las practicas se realizan bien de forma individual, bien en grupo.

- Enumerar aquellos elementos que suponen obstáculos que antes no existían

- Recoger la opinión a posteriori del alumnado, centrada en su percepción sobre las ventajas e inconvenientes de la estrategia seguida.

\section{Desarrollo de la innovación}

El trabajo colaborativo, dentro de esa mezcla que supone el trabajo autónomo con el trabajo tutorizado es algo que parece natural dentro del Espacio Europeo de Enseñanza Superior (EEES) Así, (De la Torre, 2019) nos recuerda que:

\section{El nuevo marco europeo de educación universitaria enfatiza el trabajo en grupo como una estrategia básica para la adquisición de competencias interpersonales, pues su desempeño favorece las destrezas sociales, la cooperación, la interacción y el trabajo en equipo.}

Se hace necesario conocer cuál es nuestro punto de partida. Para definirlo, nos apoyamos en las dos primeras dimensiones que definen Cabero y Marín (Cabero, Marín, 2014):

- Dimensión 1: datos descriptivos

- Dimensión 2: preferencias de aprendizaje

Obviaremos sus dimensiones 3 a 5, centradas en las capacidades técnicas y el software, pues por las características del alumnado, estudiantes del grado de informática, no suponen limitación alguna dada la población estudiada.

Nuestro punto de partida para el presente estudio es un grupo de prácticas de los considerados pequeños dentro de lo habitual en la asignatura, formado por 20 alumnos. La elección de este grupo ha sido intencional, para poder analizar de mejor modo los casos particulares, al ser su dimensión más reducida, para, en cursos posteriores (en concreto en el curso 2018-2019, ahora en marcha) ampliarlo a grupos más grandes y comprobar posibles desviaciones. Establecido un tamaño de grupo de prácticas de 4 personas, aparecen pues cinco grupos de trabajo.

\subsection{Dimensión 1: datos descriptivos}


Trabajo en grupo aplicado para la realización de las memorias de prácticas de una asignatura de corte jurídico para ingenieros.

La población de estudio estaba compuesta por un $85 \%$ de hombres y un $15 \%$ de mujeres (17 y 3 respectivamente), y con una edad media aproximada de 19 años (15 de 19, 4 de 20 y 1 de 21$)$.

\subsection{Dimensión 1: preferencias de aprendizaje}

Se les realizó una encuesta en la primera sesión, de la que se extraen los siguientes datos:

Tabla 1. Resultados de la encuesta realizada en la primera sesión

\begin{tabular}{ll}
\hline Pregunta & $\%$ \\
\hline He realizado prácticas antes de forma individual & $100 \%$ \\
\hline He realizado prácticas antes en grupo & $100 \%$ \\
\hline El trabajo en grupo es útil para aprender de los demás compañeros & $85 \%$ \\
\hline Me da la sensación de que trabajo más que los demás & $70 \%$ \\
\hline Los trabajos se hacen más rápido si todos colaboramos & $75 \%$ \\
\hline Trabajar en grupo me facilita mejorar mis habilidades sociales & $75 \%$ \\
\hline Trabajar en grupo me acerca a compartir las ideas de otras personas & $60 \%$ \\
\hline Las discusiones en grupo nunca aportan nada importante & $60 \%$ \\
\hline Trabajar en grupo empeora mis calificaciones & $60 \%$ \\
\hline No me siento cómodo trabajando con otros, pero lo veo necesario & $15 \%$ \\
\hline Me siento más cómodo trabajando solo, si pudiera escogería esa opción & $10 \%$ \\
\hline
\end{tabular}

Sobre estos resultados volvimos al final de la experiencia, para ver en qué medida podían haber cambiado las percepciones.

Por otra parte, hay una serie de elementos de partida que condicionan al docente, y que son vistos desde su prisma particular. Para ello añadimos una dimensión adicional, que denominaremos "condicionamientos docentes".

\subsection{Dimensión 3: Condicionamientos docentes}

Hay elementos técnicos y humanos a considerar. De entre los técnicos, la logística pasa por la plataforma de formación de la Universitat Politècnica de València, Poliformat, que si bien permite el trabajo en grupo y facilita la labor del docente mediante rúbricas, entregas grupales, coevaluación, y otros elementos, por otra resulta poco dinámica. Así, un grupo una vez formado y asignado a una entrega concreta (una "tarea") resulta muy difícil de romper, o de añadir, si llega un alumno rezagado en las primeras prácticas (algo muy común por problemas de matriculación) que puede quedar descolgado.

Esta casi inmovilidad de los grupos dificulta el trabajo del docente, forzándolo a establecer grupos únicos durante el curso, de forma que sean estables a lo largo de todas las entregas, 
además de ser problemático en casos de cambios o bajas de matrícula, necesidad de romper un grupo por enfrentamientos personales entre sus miembros, etc.

De entre los humanos, partiendo del trabajo de (Del Canto, Gallego et al., 2009), tuvimos en cuenta la elevada disparidad de perfiles, que van desde el alumno que no hace su trabajo que pero que se adosa en la entrega de los demás, al alumno con un elevado conocimiento de la materia que ejerce como una suerte de profesor entre sus compañeros. Es de interés subrayar dos problemas que anticipábamos y a frente los que se intentó estar prevenido: la posible falta de comunicación asertiva entre sí, que provocara malos entendidos y por tanto perjuicios para todos, algo frente a lo que se propuso llevar una bitácora de elementos pendientes y responsables de cada actividad, para minimizar perjuicios, y, por otra, la natural existencia de objetivos distintos entre los alumnos: desde el que busca tan solo aprobar, al que desea la máxima nota, pasando por el que prima ante todo su aprendizaje.

Los casos extremos, aquellos donde hay personas que no hacen su parte del trabajo, o tienen dificultades para llegar a acuerdos, o la generación de incompatibilidades que imposibilitan el trabajo conjunto no se llegaron a dar. Para casos extremos se tenía previsto un mecanismo de expulsión del grupo, cuyo primer paso discurría por un mensaje anónimo al docente, y no llegó uno solo de estos durante el desarrollo de la asignatura.

Queda un elemento por definir: cómo se crearon los grupos originales. Las dos opciones que se barajaron fue crearlos de forma aleatoria o dejar que fueran los propios alumnos los que lo constituyeran. Se optó por esta opción pues, a pesar de los problemas que se podían intuir ("amiguismos" mal entendidos que provocaran que unos trabajaran por otros, etc.) se valoró que, siendo suya la elección de compañeros, las fricciones entre miembros se minimizarían. A la luz de lo expuesto en el párrafo anterior, de forma casual o causal, se consiguió.

\subsection{Descripción del trabajo de los alumnos}

Se realizan cuatro entregas de prácticas y un examen de tipo test. La nota de este último es casi anecdótica, 0,25 puntos, y tan solo sirve para establecer una comparación, tremendamente subjetiva si se quiere, pero al menos de posible y fácil realización, con el montante final de las prácticas conseguido como trabajo en grupo (en total, otros 3,25 puntos). Esta es, como se ha dicho, tan subjetiva, que todo análsis del mismo queda fuera de la presente comunicación.

En todo momento lo que se priorizaba era el aprendizaje colaborativo, que, como nos recuerda (Glinz, 2005),

Esta técnica se refiere a la actividad que efectúan pequeños grupos de alumnos dentro de las aulas de clase; éstos se forman después de las indicaciones explicadas por el docente. Durante el inicio de la actividad y al interior del grupo, los integrantes intercambian información, tanto la que activan (conocimientos previos), como la que investigan. Posteriormente trabajan en la tarea propuesta hasta que han concluido y comprendido a fondo todos los conceptos de la temática abordada, aprendiendo así a través de la cooperación. 
Trabajo en grupo aplicado para la realización de las memorias de prácticas de una asignatura de corte jurídico para ingenieros.

Sus fundamentos, siempre según (Glinz, 2005), pasan por la siguiente relación:

- Cooperación: al apoyarse entre sí los alumnos, que deben considerar que su propio éxito depende del éxito del grupo.

- Responsabilidad: cada uno es responsable del trabajo que les fue asignado por el grupo, pero al depender el resultado de la suma de las partes, todo el grupo permanece involucrado en la tarea de cada uno.

- Comunicación: se retroalimentan unos a otros, analizan los resultados de cada integrante buscando resultados de mejor calidad.

- Trabajo en equipo: desarrollan habilidades de comunicación, liderazgo, confianza, …

- Autoevaluación: el equipo se fija las metas y realiza una evaluación para rectificar si es preciso.

Así, la división de los trabajos a realizar pasa por el siguiente esquema de tiempos:

Tabla 2. Esquema de tiempos

\begin{tabular}{|c|c|c|c|}
\hline Entrega & Tareas a realizar & Sesiones & El profesor: \\
\hline 1 & $\begin{array}{l}\text { Estudio de perfiles profesionales } \\
\text { Localización de normativa } \\
\text { técnica } \\
\text { Elaboración de informe }\end{array}$ & $\begin{array}{l}1,2,3,4 \\
(1,5 * 4=6 \\
\text { horas })\end{array}$ & $\begin{array}{l}\text { Presentación del caso. (30 } \\
\text { min) } \\
\text { Aporta la información inicial } \\
(30 \mathrm{~min}) \\
\text { Formación de grupos y primer } \\
\text { contacto con ellos ( } 30 \mathrm{~min}) \\
\text { Instrucciones sobre el manejo } \\
\text { de herramientas (30 min) } \\
\text { Tutorización de las tareas (3h) } \\
\text { Revisión de los entregables } \\
\text { antes de su envío }(1 \mathrm{~h})\end{array}$ \\
\hline 2 & $\begin{array}{l}\text { Localización de sentencias y } \\
\text { legislación } \\
\text { Elaboración de informe }\end{array}$ & $\begin{array}{l}5,6 \\
(1,5 * 2=3 \\
\text { horas })\end{array}$ & $\begin{array}{l}\text { Presentación del caso. } \\
\text { min) } \\
\text { Instrucciones sobre el manejo } \\
\text { de herramientas ( } 30 \text { min) } \\
\text { Tutorización de las tareas (1,5 } \\
\text { h) } \\
\text { Revisión de los entregables } \\
\text { antes de su envío (30 min) }\end{array}$ \\
\hline
\end{tabular}




\begin{tabular}{|c|c|c|c|}
\hline Entrega & Tareas a realizar & Sesiones & EI profesor: \\
\hline 3 & $\begin{array}{l}\text { Elaboración de dictamen } \\
\text { pericial } \\
\text { Evaluación de dictamen pericial } \\
\text { (Corrección) }\end{array}$ & $\begin{array}{l}7,8 \\
(1,5 * 2=3 \\
\text { horas })\end{array}$ & $\begin{array}{l}\text { Presentación del caso. (30 } \\
\text { min) } \\
\text { Instrucciones sobre el manejo } \\
\text { de herramientas ( } 30 \mathrm{~min}) \\
\text { Tutorización de las tareas (2 } \\
\text { h) } \\
\text { (incluyendo corrección) }\end{array}$ \\
\hline 4 & $\begin{array}{l}\text { Localización de normas técnicas } \\
\text { Elaboración de informe }\end{array}$ & $\begin{array}{l}9,10 \\
(1,5 * 2=3 \\
\text { horas })\end{array}$ & $\begin{array}{l}\text { Presentación del caso. (30 } \\
\text { min) } \\
\text { Tutorización de las tareas ( } 2 \\
\text { h) } \\
\text { Revisión de los entregables } \\
\text { antes de su envío ( } 30 \mathrm{~min})\end{array}$ \\
\hline
\end{tabular}

\section{Resultados}

Planteábamos que nuestros objetivos eran

- Medir la mejora en la tasa de retrasos y de ausencias en las entregas de las memorias

- Recoger la opinión apriorística del alumnado, que ya ha cursado asignaturas donde las practicas se realizan bien de forma individual, bien en grupo.

- Enumerar aquellos elementos que suponen obstáculos que antes no existían

- Recoger la opinión a posteriori del alumnado, centrada en su percepción sobre las ventajas e inconvenientes de la estrategia seguida.

El primer objetivo es de fácil revisión: la mejora ha sido drástica. Se ha pasado de un $10 \%$ de abandono y un 30\% de retraso a una ausencia de abandono (incluyendo hipotéticos casos de desmatriculación en la asignatura, pues no se dieron), a un $10 \%$ de retraso, que se traduce en la entrega tardía de una misma práctica, la segunda, en dos grupos, con el elemento externo a considerar de la proximidad de la entrega con la semana de exámenes, lo que siempre es una lógica distorsión en el trabajo del alumno.

El segundo objetivo queda resumido en la tabla 1.

El tercer objetivo pasa por esos elementos técnicos a los que aludíamos en el apartado 1.3. Puede observarse si se quiere una subjetividad en la afirmación, pero el trabajo con las herramientas de entrega de tareas y gestión de grupos de la plataforma Poliformat han supuesto un buen quebradero de cabeza para el docente. La única solución que se puede atisbar a esto pasa por la propuesta de soluciones desde los docentes a los gestores de la misma. 
Trabajo en grupo aplicado para la realización de las memorias de prácticas de una asignatura de corte jurídico para ingenieros.

El cuarto y último objetivo pasó por una encuesta realizada tras la última entrega de las prácticas, cuyos resultados pueden verse en la tabla 3. Como puede verse, es muy simple, y se basa en verificar si ha sufrido algún cambio la percepción de los alumnos con respecto a la primera encuesta realizada, además de preguntar por elementos concretos que puedan haber sido ventajas o inconvenientes para ellos en esta experiencia.

Tabla 3. Resultados de la encuesta realizada en la última sesión

\begin{tabular}{|c|c|}
\hline Pregunta & $\%$ \\
\hline $\begin{array}{l}\text { Considero que he aprendido o reforzado mis conocimientos técnicos gracias } \\
\text { a mis compañeros }\end{array}$ & $95 \%$ \\
\hline Creo que he trabajado más que mis compañeros de grupo & $15 \%$ \\
\hline Creo que merezco más nota que mis compañeros de grupo & $10 \%$ \\
\hline $\begin{array}{l}\text { Al trabajar en grupo, se han realizado los trabajos con a tiempo para las } \\
\text { entregas. }\end{array}$ & $20 \%$ \\
\hline Trabajar en grupo me ha permitido mejorar mis habilidades sociales & $100 \%$ \\
\hline Trabajar en grupo me acerca a compartir las ideas de otras personas & $100 \%$ \\
\hline Las discusiones en grupo nunca aportan nada importante & $5 \%$ \\
\hline $\begin{array}{l}\text { Las explicaciones del profesor al grupo permiten que estas sean más } \\
\text { profundas, frente a las explicaciones individuales. }\end{array}$ & $85 \%$ \\
\hline $\begin{array}{l}\text { Explicando cosas a mis compañeros de grupo, tengo la sensación de } \\
\text { aprender más y mejor. }\end{array}$ & $85 \%$ \\
\hline $\begin{array}{l}\text { Las herramientas disponibles en Poliformat me han resultado cómodas y } \\
\text { útiles }\end{array}$ & $50 \%$ \\
\hline $\begin{array}{l}\text { He aprendido a manejar las herramientas externas (Aranzadi, AENOR...) } \\
\text { fundamentalmente gracias a las explicaciones del profesor en el aula. }\end{array}$ & $20 \%$ \\
\hline $\begin{array}{l}\text { He aprendido a manejar las herramientas externas (Aranzadi, AENOR...) } \\
\text { fundamentalmente gracias a los vídeos docentes explicativos de las mismas. }\end{array}$ & $10 \%$ \\
\hline $\begin{array}{l}\text { He aprendido a manejar las herramientas externas (Aranzadi, AENOR...) } \\
\text { fundamentalmente gracias a mis compañeros de grupo. }\end{array}$ & $70 \%$ \\
\hline
\end{tabular}

\section{Conclusiones}

La simple comparación de las tablas 1 y 3 , donde se percibe el cambio en positivo de la percepción de los alumnos, y el dato objetivo de la reducción absoluta de abandonos y significativa de retrasos nos permiten afirmar que el cambio ha valido la pena. Cabe indicar que no se hace alusión alguna al resultado académico pues, excepto algún cambio muy leve 
en los decimales de los porcentajes, las tasas de suspensos, aprobados, notables y sobresalientes, se mantienen sin variación.

Queda, como elementos pendientes para el curso actual y siguientes, perfilar un mejor reparto de tiempos, ya que como se ve en la tabla $\mathrm{n}^{\circ} 2$ resulta muy desigual (producto de haber heredado una estructura de prácticas basada en entregas individuales) y realizar un listado exhaustivo de mejoras propuesta en la gestión de grupos y entregas de tareas de la plataforma Poliformat, para evitar problemas tanto a docentes como a discentes.

\section{Referencias}

CABERO, Julio; Marín, Verónica (2014) "Posibilidades educativas de las redes sociales y el trabajo en grupo. Percepciones de los alumnos universitarios". Comunicar Revista Cientifica de Educomunicación no 42, v. XXI, 2014, pp 165-172. <http://dx.doi.org/10.3916/C42-2014-16> [Consulta: 1 de marzo de 2019]

DE LA TORRE-LASO, Jesús, (2019) “ La retroalimentación evaluativa o feedback para los trabajos en grupo como estrategia de acción tutorial en la universidad". Educación, vol. 43, núm. 1, 2019. <https://doi.org/10.15517/revedu.v43i1.30062> [Consulta: 1 de marzo de 2019]

DEL CANTO, Pablo; GALLEGO, Isabel; LÓPEZ, José Manuel et al. (2009). «Conflictos en el trabajo en grupo: cuatro casos habituales " Revista de Formación e Innovación Educativa $\begin{array}{lllllll}\text { Universitaria. } & \text { Vol. } & 2, & \mathrm{~N}^{\mathrm{o}} & 4, & \text { pp. } & 211-226<\end{array}$ http://refiedu.webs.uvigo.es/Refiedu/Vol2_4/REFIEDU_2_4_4.pdf $>$ [Consulta: 1 de marzo de 2019]

GLINZ FEREZ, Patricia E. (2005). «Un acercamiento al trabajo colaborativo». Revista Iberoamericana De Educación, 36(7), pp. 1-14. <https://rieoei.org/RIE/article/view/2927> [Consulta: 1 de marzo de 2019]

OLTRA GUTIÉRREZ, Juan V. (2018). « Método del caso como medio de evaluación dentro del modelo FLIP-Teaching. Una experiencia con una asignatura de corte jurídico para ingenieros ». IN-RED 2018. IV Congreso Nacional de Innovación Educativa y Docencia en Red. Editorial Universitat Politècnica de València. Pp. 492-501. <http://hdl.handle.net/10251/112370> [Consulta: 1 de marzo de 2019] 